\title{
GREEN FUNCTION FOR ANALOGUE OF ROBIN PROBLEM FOR POLYHARMONIC EQUATION
}

\author{
B.Kh. TURMETOV
}

\begin{abstract}
We propose a method of constructing the Green function for some boundary value problems for a polyharmonic equation in a multi-dimensional unit ball. The considered problem are analogues of the Robin problem for an inhomogeneous polyharmonic equation. For studying the solvability of these problems in the class of smooth in a ball functions, we first provide the properties of integral-differential operators. Then, employing these properties, the considered problems are reduced to an equivalent Dirichlet problem with a special right hand side. Using then known statements on the Dirichlet problem, for the main problems we prove the unique solvability theorems. We also obtain integral representations for solutions of these problems via the solutions of the Dirichlet problem. Employing the explicit form of the Green function, we find an integral representation of the Dirichlet problem with a special right hand side. The obtained integral representation then is used to construct the Green function for analogues of Robin problems. We also provide an approach for constructing the Green function for other main problems. In order to do this, we study the differential properties of the fundamental solution of the polyharmonic operator. The obtained properties of the fundamental solutions are applied for studying the properties of the Green function for the Dirichlet problem. We construct the representations of the Green function for analogues of the Robin problem. While finding the Green functions for these problems, we employ essentially the form of the Green function for the Dirichlet problem for the polyhgarmonic equation. Namely, the Green function of these problems is represented as the sum of the Green function for the Dirichlet problem and some integral term. The obtained results are in agreement with the known results for the Laplace operator.
\end{abstract}

Keywords: polyharmonic equation, boundary value problem, Dirichlet problem, analogue of Robin problem, Green function, integral representation.

Mathematics Subjects Classifications: 35J40, 31B30

\section{INTRODUCTION}

Let $\Omega=\left\{x \in \mathbb{R}^{n}:|x|<1\right\}$ be an $n$-dimensional unit ball, $n \geqslant 2, \partial \Omega=\left\{x \in \mathbb{R}^{n}:|x|=1\right\}$ be a unit sphere, $\nu$ be an outward normal to $\partial \Omega$. The Dirichlet problem for an inhomogeneous polyharmonic equation

$$
(-\Delta)^{m} v(x)=F(x), \quad x \in \Omega, \quad \frac{\partial^{k} v(x)}{\partial \nu^{k}}=0, \quad x \in \partial \Omega, \quad k=0,1, \ldots, m-1, \quad m \geqslant 1,
$$

B.Kh. Turmetov, Green function for analogue of Robin problem for polyharmonic equaTION.

This work is financially supported by a grant of Ministry of Education and Science of the Republic of Kazakhstan (no. AP05131268).

Submityted July 14, 2018. 
in the domain $\Omega$ is a classical and well-studied problem.

If the function $F(x)$ is smooth, then problem (1) is uniquely solvable and is represented by means of the Green function:

$$
v(x)=\int_{\Omega} G_{D, m}(x, y) F(y) d y .
$$

The Green function for the Dirichlet problem was constructed explicitly in various ways in works [1]-[5]. For instance, it was shown in [1] that the function $G_{D, m}(x, y)$ reads as

$$
G_{D, m}(x, y)=K_{m, n}|x-y|^{2 m-n} \int_{1}^{g(x, y)}\left(t^{2}-1\right)^{m-1} t^{1-n} d t
$$

where

$$
g(x, y)=\frac{1}{|x-y|}|x| y\left|-\frac{y}{|x|}\right|, \quad K_{m, n}=\frac{1}{4^{m-1}((m-1) !)^{2} n e_{n}}, \quad e_{n}=\frac{\pi^{n / 2}}{\Gamma\left(1+\frac{n}{2}\right)} .
$$

It is easy to obtain the following representation for the coefficient $K_{m, n}$, see, for instance, [6]:

$$
K_{m, n}=\frac{1}{4^{m-1}((m-1) !)^{2} \omega_{n}},
$$

where $\omega_{n}=2 \pi^{n / 2} / \Gamma(n / 2)$ is the area of the unit sphere.

In the case $m=1$, that is, for the Poisson equation, apart of the Dirichlet problem, the Robin problem is also classical and well-studied:

$$
-\Delta u(x)=f(x), \quad x \in \Omega, \quad \frac{\partial u(x)}{\partial \nu}+a u(x)=0, \quad x \in \partial \Omega,
$$

where $0<a$ is a real number. The Green function for this problem was explicitly found in works [7]- 9].

Let

$$
r=|x|, \quad r \frac{\partial}{\partial r}=\sum_{j=1}^{n} x_{j} \frac{\partial}{\partial x_{j}}
$$

We consider the operators

$$
\Gamma_{a}=\left(r \frac{\partial}{\partial r}+a\right), \quad \Gamma_{a}^{(k)}=\left(r \frac{\partial}{\partial r}+a\right)^{k} \equiv \underbrace{\Gamma_{a} \cdot \ldots \cdot \Gamma_{a}}_{k}, \quad k \geqslant 2 .
$$

The properties and applications of the operators like $\Gamma_{a}^{(k)}$ in the class of harmonic functions were studied in [10], [11].

In the present work we provide a method for constructing the Green function for the following analogue of the Robin problem:

$$
(-\Delta)^{m} u(x)=f(x), \quad x \in \Omega, \quad \Gamma_{a}^{(k)}[u](x)=0, \quad x \in \partial \Omega, \quad k=1,2, \ldots, m .
$$

A solution to this problem is a function $u(x) \in C^{2 m}(\Omega) \cap C^{m}(\bar{\Omega})$ satisfying the conditions of problem (5) in a classical sense. 


\section{Auxiliary statements}

In this section we provide some properties of the solutions of Dirichlet problem (1), present a method of reducing problem (5) to an auxiliary Dirichlet problem, and prove the unique solvability of this problem.

We consider an operator

$$
\Gamma_{a}^{-1}[u](x)=\int_{0}^{1} s^{a-1} u(s x) d s .
$$

The following lemma is true [10], [12].

Lemma 1. If $a>0$ and $u(x)$ is a smooth function in the domain $\bar{\Omega}$, then the identities

$$
\Gamma_{a}\left[\Gamma_{a}^{-1}[u]\right](x)=\Gamma_{a}^{-1}\left[\Gamma_{a}[u]\right](x)=u(x)
$$

hold for each $x \in \bar{\Omega}$.

Lemma 2. Let $f \in C^{\lambda+1}(\bar{\Omega}), 0<\lambda<1$. Then problem (5) is uniquely solvable and is represented as

$$
u(x)=\int_{0}^{1} s^{a-1} v(s x) d s \equiv \Gamma_{a}^{-1}[v](x),
$$

where $v(x)$ is the solution to Dirichlet problem (1) with the function

$$
F(x)=\left(r \frac{\partial}{\partial r}+2 m+a\right) f(x) .
$$

Proof. Suppose that $u(x)$ is a solution to problem (5). We apply the operator $\Gamma_{a}$ to this function and we denote $\Gamma_{a}[u](x)=v(x)$. Let us find conditions for the function $v(x)$. Since

$$
r \frac{\partial}{\partial r}=\sum_{j=1}^{n} x_{j} \frac{\partial}{\partial x_{j}}, \Delta\left(\sum_{j=1}^{n} x_{j} \frac{\partial u(x)}{\partial x_{j}}\right)=\left(r \frac{\partial}{\partial r}+2\right) \Delta u(x)=\Gamma_{2}[\Delta u](x),
$$

we get

$$
(-\Delta)^{m} v(x)=\left(r \frac{\partial}{\partial r}+a+2 m\right)(-\Delta)^{m} u(x)=\Gamma_{2 m+a}[f](x) \equiv F(x) .
$$

For each $x \in \partial \Omega$ we have

$$
\begin{aligned}
0 & =\left.\left(r \frac{\partial}{\partial r}+a\right) u(x)\right|_{\partial \Omega}=\left.v(x)\right|_{\partial \Omega}, \quad 0=\left.\left(r \frac{\partial}{\partial r}+a\right)^{2} u(x)\right|_{\partial \Omega} \\
& =\left.\left(r \frac{\partial}{\partial r}+a\right) v(x)\right|_{\partial \Omega}=\left.\frac{\partial}{\partial \nu} v(x)\right|_{\partial \Omega}+\left.a v(x)\right|_{\partial \Omega} .
\end{aligned}
$$

Therefore,

Then

$$
\left.v(x)\right|_{\partial \Omega}=0,\left.\quad r \frac{\partial v(x)}{\partial r}\right|_{\partial \Omega}=\left.\frac{\partial v(x)}{\partial \nu}\right|_{\partial \Omega}=0 .
$$

On the other hand,

$$
\left.\left(r \frac{\partial}{\partial r}+a\right)^{2} v(x)\right|_{\partial \Omega}=\left.\left(r \frac{\partial}{\partial r}+a\right)^{3} u(x)\right|_{\partial \Omega}=0
$$

$$
\left.\left(r \frac{\partial}{\partial r}+a\right)^{2} v(x)\right|_{\partial \Omega}=\left.\left(r^{2} \frac{\partial^{2}}{\partial r^{2}}+(2 a+1) r \frac{\partial}{\partial r}+a^{2}\right) v(x)\right|_{\partial \Omega}
$$


Then

$$
=\left.\frac{\partial^{2} v(x)}{\partial \nu^{2}}\right|_{\partial \Omega}+\left.(2 a+1) \frac{\partial v(x)}{\partial \nu}\right|_{\partial \Omega}+\left.a^{2} v(x)\right|_{\partial \Omega}=\left.\frac{\partial^{2} v(x)}{\partial \nu^{2}}\right|_{\partial \Omega} .
$$

$$
\left.\frac{\partial^{2} v(x)}{\partial \nu^{2}}\right|_{\partial \Omega}=0
$$

In the same way we show that for each $k=3,4, \ldots, m-1$ the identities hold:

$$
\left.\frac{\partial^{k} v(x)}{\partial \nu^{k}}\right|_{\partial \Omega}=0
$$

Hence, for the function $v(x)=\Gamma_{a}[u](x)$ we obtain Dirichlet problem (1) with the function $F(x)=\Gamma_{2 m+a}[f](x)$. If $f(x) \in C^{\lambda+1}(\bar{\Omega})$, then $F(x) \in C^{\lambda}(\bar{\Omega})$, and solution to problem (1) exists, is unique and can be represented as (2).

Then by identity (6) we find

$$
u(x)=\int_{0}^{1} s^{a-1} v(s x) d s
$$

and representation (7) holds true.

Let a function $v(x)$ solves problem (1) with the function $F(x)=\Gamma_{2 m+a}[f](x)$. We are going to show that the function $u(x)=\Gamma_{a}^{-1}[v](x)$ satisfies all conditions of problem (5). Indeed, since

$$
(-\Delta)^{m} \Gamma_{a}^{-1}[v](x)=\int_{0}^{1} s^{a+2 m-1} F(s x) d s=\Gamma_{a+2 m}^{-1}[F](x), \quad F(x)=\Gamma_{2 m+a}[f](x),
$$

by identity (6) we get

$$
(-\Delta)^{m} u(x)=(-\Delta)^{m} \Gamma_{a}^{-1}[v](x)=\Gamma_{2 m+a}^{-1}\left[\Gamma_{2 m+a}[f]\right](x)=f(x) .
$$

In the integral $\int_{0}^{1} s^{a-1} v(s x) d s$ we make the change of the variables $s r=\xi$. Then $u(x)$ is represented as

$$
u(x)=r^{-a} \int_{0}^{r} \xi^{a-1} v(\xi \theta) d \xi, \quad \theta=\frac{x}{|x|}
$$

This yields

$$
\begin{aligned}
\left.\Gamma_{a}[u](x)\right|_{\partial \Omega} & =\left.\left(r \frac{\partial}{\partial r}+a\right) u(x)\right|_{\partial \Omega} \\
& =\left.\left(-a r^{-a} \int_{0}^{r} \xi^{a-1} v(\xi \theta) d s+v(x)+a r^{-a} \int_{0}^{r} \xi^{a-1} v(\xi \theta) d s\right)\right|_{r=1}=\left.v(x)\right|_{\partial \Omega}=0, \\
\left.\Gamma_{a}^{(2)}[u](x)\right|_{\partial \Omega} & =\left.\left(r \frac{\partial}{\partial r}+a\right) v(x)\right|_{\partial \Omega}=\left.\frac{\partial v(x)}{\partial \nu}\right|_{\partial \Omega}+\left.a v(x)\right|_{\partial \Omega}=0 .
\end{aligned}
$$

Since for each $k=3,4, \ldots, m$ the identities hold [13]

$$
\begin{aligned}
\left.\left(r \frac{\partial}{\partial r}+a\right)^{k} u(x)\right|_{\partial \Omega} & =\left.\left(r \frac{\partial}{\partial r}+a\right)^{k-1} v(x)\right|_{\partial \Omega} \\
& =\left.\sum_{j=0}^{k-1} C_{k-1}^{j} a^{k-1-j}\left(r \frac{\partial}{\partial r}\right)^{j} v(x)\right|_{\partial \Omega}=\left.\sum_{j=0}^{k-1} C_{k-1}^{j} a^{k-1-j} \sum_{i=0}^{j} d_{i, j} \frac{\partial^{i} v(x)}{\partial \nu^{i}}\right|_{\partial \Omega}=0,
\end{aligned}
$$


where $d_{i, j}$ are some constants, boundary conditions in problem (5) are also satisfied.

Lemma 3. Let the function $F(x)$ read as

$$
F(x)=\left(r \frac{\partial}{\partial r}+a+2 m\right) f(x) .
$$

Then solution of Dirichlet problem (1) is represented as

$$
v(x)=\int_{\Omega}\left(2 m+a-n-\rho \frac{\partial}{\partial \rho}\right) G_{D, m}(x, y) f(y) d y .
$$

Proof. We consider the function

$$
I_{j}=\int_{\Omega} G_{D, m}(x, y) y_{j} \frac{\partial}{\partial y_{j}} f(y) d y, \quad j=1,2, \ldots, n .
$$

Employing the approach of [14], we integrate by parts in $I_{j}$ taking into consideration the property of the Green function $\left.G_{D, m}(x, y)\right|_{|y|=1}=0$ and we get

$$
\begin{aligned}
I_{j}= & \int_{\Omega} y_{j} G_{D, m}(x, y) \frac{\partial}{\partial y_{j}} f(y) d y=\int_{\partial \Omega} y_{j} G_{D, m}(x, y) f(y) \cos \left(\widehat{\nu_{y} y_{j}}\right) d S_{y} \\
& -\int_{\Omega} \frac{\partial}{\partial y_{j}}\left[y_{j} G_{D, m}(x, y)\right] f(y) d y=-\int_{\Omega}\left(1+y_{j} \frac{\partial}{\partial y_{j}}\right) G_{D, m}(x, y) f(y) d y .
\end{aligned}
$$

Here $\widehat{\nu_{y} y_{j}}$ is an angle between $\nu_{y}$ and $y_{j}$. Then

$$
\begin{aligned}
\int_{\Omega} G_{D, m}(x, y) \rho \frac{\partial}{\partial \rho} f(y) d y & =\int_{\Omega} G_{D, m}(x, y) \sum_{j=1}^{n} y_{j} \frac{\partial}{\partial y_{j}} f(y) d y \\
& =-\int_{\Omega} \sum_{j=1}^{n} \frac{\partial}{\partial y_{j}}\left[y_{j} G_{D, m}(x, y)\right] f(y) d y \\
& =-\int_{\Omega}\left(n+\rho \frac{\partial}{\partial \rho}\right) G_{D, m}(x, y) f(y) d y
\end{aligned}
$$

Hence, the identity holds:

$$
\int_{\Omega} G_{D, m}(x, y)\left(2 m+a+\rho \frac{\partial}{\partial \rho}\right) f(y) d y=\int_{\Omega}\left(2 m+a-n-\rho \frac{\partial}{\partial \rho}\right) G_{D, m}(x, y) f(y) d y .
$$

This completes the proof.

Corollary 1. Let $f(x) \in C^{\lambda+1}(\bar{\Omega})$. Then problem (5) is uniquely solvable and the solution can be represented as

$$
u(x)=\int_{\Omega}\left[\int_{0}^{1} s^{a-1}\left(a+2 m-n-\rho \frac{\partial}{\partial \rho}\right) G_{D, m}(s x, y)\right] f(y) d y .
$$




\section{Construction of Green function}

In this section we construct the Green function for the main problem. We denote

$$
\rho \frac{\partial}{\partial \rho}=\sum_{j=1}^{n} y_{j} \frac{\partial}{\partial y_{j}}
$$

We begin with some auxiliary statements.

Lemma 4. The function

$$
g(x, y)=\frac{1}{|x-y|}|x| y\left|-\frac{y}{|x|}\right|
$$

satisfies the identity

$$
|x-y|^{2 m-n}\left(g^{2}(x, y)-1\right)^{m-1} g^{2-n}(x, y)=\frac{\left(1-|x|^{2}\right)^{m-1}\left(1-|y|^{2}\right)^{m-1}}{|x| y\left|-\frac{y}{|y|}\right|^{n-2}} .
$$

Proof. Since

$$
|x| y\left|-\frac{y}{|y|}\right|^{2}-|x-y|^{2}=\left(1-|x|^{2}\right)\left(1-|y|^{2}\right),
$$

the definition of $g(x, y)$ yields

$$
\begin{aligned}
|x-y|^{2 m-n}\left(g^{2}(x, y)-1\right)^{m-1} g^{2-n}(x, y)= & |x-y|^{2 m-n} \frac{\left(1-|x|^{2}\right)^{m-1}\left(1-|y|^{2}\right)^{m-1}}{|x-y|^{2 m-2}} \\
& \cdot \frac{|x-y|^{n-2}}{|x| y\left|-\frac{y}{|y|}\right|^{n-2}}=\frac{\left(1-|x|^{2}\right)^{m-1}\left(1-|y|^{2}\right)^{m-1}}{|x| y\left|-\frac{y}{|y|}\right|^{n-2}} .
\end{aligned}
$$

Lemma 5. The identities hold:

$$
\begin{aligned}
& \left(\rho \frac{\partial}{\partial \rho}+s \frac{d}{d s}\right)|s x-y|^{2 m-n}=(2 m-n)|s x-y|^{2 m-n} \\
& \left(\rho \frac{\partial}{\partial \rho}+s \frac{d}{d s}\right) g(s x, y)=g(s x, y) \frac{s^{2}|x|^{2}|y|^{2}-1}{|s x| y\left|-\frac{y}{|y|}\right|^{2}}
\end{aligned}
$$

The proof is complete.

Proof. Applying successively the operators $\rho \frac{\partial}{\partial \rho}$ and $s \frac{d}{d s}$ to the function $|s x-y|^{2 m-n}$, we get

$$
\begin{aligned}
\left(\rho \frac{\partial}{\partial \rho}+s \frac{d}{d s}\right)|s x-y|^{2 m-n}= & (2 m-n)|s x-y|^{2(m-1)-n}\left(|y|^{2}-s(x, y)\right) \\
& +(2 m-n)|s x-y|^{2(m-1)-n}\left(s^{2}|x|^{2}-s(x, y)\right) \\
= & (2 m-n)|s x-y|^{2 m-n} \frac{|y|^{2}-s(x, y)+s^{2}|x|^{2}-s(x, y)}{|s x-y|^{2}} \\
= & (2 m-n)|s x-y|^{2 m-n} .
\end{aligned}
$$

This proves identity (11).

We have

$$
\left(\rho \frac{\partial}{\partial \rho}+s \frac{d}{d s}\right) g(s x, y)=|s x-y|^{-1} \cdot\left(\rho \frac{\partial}{\partial \rho}+s \frac{d}{d s}\right)|s x| y\left|-\frac{y}{|y|}\right|
$$




$$
\begin{aligned}
& +|s x| y\left|-\frac{y}{|y|}\right|\left(\rho \frac{\partial}{\partial \rho}+s \frac{d}{d s}\right)\left(|s x-y|^{-1}\right) \\
= & |s x-y|^{-1} \cdot 2 \frac{s^{2}|x|^{2}|y|^{2}-s(x, y)}{|s x| y\left|-\frac{y}{|y|}\right|} \\
& -|s x| y\left|-\frac{y}{|y|}\right||s x-y|^{-3}\left(|y|^{2}-s(x, y)+\left|s^{2} y\right|^{2}-s(x, y)\right) \\
= & \frac{1}{|s x-y|} \cdot 2 \frac{s^{2}|x|^{2}|y|^{2}-s(x, y)}{|s x| y\left|-\frac{y}{|y|}\right|}-\frac{|s x| y\left|-\frac{y}{|y|}\right|}{|s x-y|} \\
= & \left.\frac{|s x| y\left|-\frac{y}{|y|}\right|}{|s x-y|}\right]=g(s x, y) \frac{s^{2}|x|^{2}|y|^{2}-1}{|s x| y\left|-\frac{y}{|y|}\right|^{2} .} \\
& \cdot\left[\frac{2 s^{2}|x|^{2}|y|^{2}-2 s(x, y)}{|s x| y\left|-\frac{y}{|y|}\right|^{2}}-1\right]
\end{aligned}
$$

This proves identity 12 .

Lemma 6. The identity holds

$$
\begin{aligned}
\left(\rho \frac{\partial}{\partial \rho}+s \frac{d}{d s}\right) G_{D, m}(s x, y)= & (2 m-n) G_{D, m}(s x, y) \\
& -K_{m, n}\left(1-s^{2}|x|^{2}\right)^{m-1}\left(1-|y|^{2}\right)^{m-1} \frac{1-s^{2}|x|^{2}|y|^{2}}{|s x| y\left|-\frac{y}{|y|}\right|^{n}},
\end{aligned}
$$

where $K_{m, n}$ is defined by identity (4).

Proof. Employing the representation for the function $G_{D, m}(x, y)$, we obtain

$$
\begin{aligned}
\rho \frac{\partial}{\partial \rho} G_{D, m}(s x, y)= & K_{m, n} \int_{1}^{g(s x, y)}\left(t^{2}-1\right)^{m-1} t^{1-n} d t \rho \frac{\partial}{\partial \rho}|s x-y|^{2 m-n} \\
& +K_{m, n}|s x-y|^{2 m-n}\left(g^{2}-1\right)^{m-1} g^{1-n} \rho \frac{\partial}{\partial \rho} g(s x, y) .
\end{aligned}
$$

In the same way,

$$
\begin{aligned}
s \frac{d}{d s} G_{D, m}(s x, y)= & K_{m, n} \int_{1}^{g(s x, y)}\left(t^{2}-1\right)^{m-1} t^{1-n} d t s \frac{d}{d s}|s x-y|^{2 m-n} \\
& +K_{m, n}|s x-y|^{2 m-n}\left(g^{2}-1\right)^{m-1} g^{1-n} s \frac{d}{d s} g(s x, y) .
\end{aligned}
$$

Hence,

$$
\begin{aligned}
\left(\rho \frac{\partial}{\partial \rho}+s \frac{d}{d s}\right) G_{D, m}(s x, y)= & K_{m, n} \int_{1}^{g(s x, y)}\left(t^{2}-1\right)^{m-1} t^{1-n} d t\left(\rho \frac{\partial}{\partial \rho}+s \frac{d}{d s}\right)|s x-y|^{2 m-n} \\
& +K_{m, n}|s x-y|^{2 m-n}\left(g^{2}-1\right)^{m-1} g^{1-n}\left(\rho \frac{\partial}{\partial \rho}+s \frac{d}{d s}\right) g(s x, y) .
\end{aligned}
$$


Employing identities (11), (12) and (10), we finally obtain

$$
\begin{aligned}
\left(\rho \frac{\partial}{\partial \rho}+s \frac{d}{d s}\right) G_{D, m}(s x, y)= & K_{m, n}(2 m-n)|s x-y|^{2 m-n} \int_{1}^{g(s x, y)}\left(t^{2}-1\right)^{m-1} t^{1-n} d t \\
& +K_{m, n}|s x-y|^{2 m-n}\left(g^{2}-1\right)^{m-1} g^{1-n} g(s x, y) \frac{s^{2}|x|^{2}|y|^{2}-1}{|s x| y\left|-\frac{y}{|y|}\right|^{2}} \\
= & (2 m-n) G_{D, m}(s x, y) \\
& -K_{m, n}\left(1-s^{2}|x|^{2}\right)^{m-1}\left(1-|y|^{2}\right)^{m-1} \frac{1-s^{2}|x|^{2}|y|^{2}}{|s x| y\left|-\frac{y}{|y|}\right|^{n}}
\end{aligned}
$$

The proof is complete.

We proceed to a main statement on the Green function for problem (5).

Theorem 1. Let $a>0,0<\lambda<1$ and $f \in C^{\lambda+1}(\bar{\Omega})$. Then the solution of problem (5) can be represented as

$$
u(x)=\int_{\Omega} G_{a}(x, y) f(y) d y,
$$

where the Green function $G_{a}(x, y)$ reads as

$$
G_{a}(x, y)=G_{D, m}(x, y)+K_{m, n}\left(1-|y|^{2}\right)^{m-1} \int_{0}^{1} s^{a-1} \frac{\left(1-s^{2}|x|^{2}\right)^{m-1}\left(1-s^{2}|x|^{2}|y|^{2}\right)}{|s x| y\left|-\frac{y}{|y|}\right|^{n}} d s .
$$

Proof. Since $f \in C^{\lambda+1}(\bar{\Omega})$, by Lemma 2, problem (5) is uniquely solvable and the solution is given by (7), where $v(x)$ is the solution of Dirichlet problem (1) with the function

$$
F(x)=\left(r \frac{\partial}{\partial r}+2 m+a\right) f(x) .
$$

By Corollary 1, the solution of problem (5) can be represented as (9), that is,

$$
u(x)=\int_{\Omega}\left[\int_{0}^{1} s^{a-1}\left(a+2 m-n-\rho \frac{\partial}{\partial \rho}\right) G_{D, m}(s x, y)\right] f(y) d y .
$$

We denote

$$
G_{a}(x, y)=\int_{0}^{1} s^{a-1}\left(a+2 m-n-\rho \frac{\partial}{\partial \rho}\right) G_{D, m}(s x, y) d s .
$$

Let us study the function

$$
\left(a+2 m-n-\rho \frac{\partial}{\partial \rho}\right) G_{D, m}(s x, y)
$$

By identity 13 we have

$$
\begin{aligned}
\left(a+2 m-n-\rho \frac{\partial}{\partial \rho}\right) G_{D, m}(s x, y)= & \left(s \frac{d}{d s}+a\right) G_{D, m}(s x, y) \\
& +K_{m, n}\left(1-|y|^{2}\right)^{m-1} \frac{\left(1-s^{2}|x|^{2}\right)^{m-1}\left(1-s^{2}|x|^{2}|y|^{2}\right)}{|s x| y\left|-\frac{y}{|y|}\right|^{n}} .
\end{aligned}
$$


On the other hand,

$$
\int_{0}^{1} s^{a-1}\left(s \frac{d}{d s}+a\right) G_{D, m}(s x, y) d s=\int_{0}^{1} \frac{d}{d s}\left[s^{a} G_{D, m}(s x, y)\right] d s=G_{D, m}(x, y) .
$$

This implies identity 15$)$ for the function $G_{a}(x, y)$ and representation (14) for the solution of problem (5).

Corollary 2. As $m=1$, the Green function of the Robin problem for the Poisson equation can be represented as

$$
G_{a}(x, y)=G_{D, 1}(x, y)+\frac{1}{\omega_{n}} \int_{0}^{1} s^{a-1} \frac{1-s^{2}|x|^{2}|y|^{2}}{|s x| y\left|-\frac{y}{|y|}\right|^{n}} d s .
$$

In the case $n=2$, this corollary was obtained in work [7], while the case $n>2$ was resolved in [8].

We proceed to a generalization of problem (5). Let $a_{j}, j=1,2, \ldots, m$, be positive real numbers. We introduce the notations

$$
\Gamma_{a_{1}, a_{2}, \ldots, a_{\ell}}^{(\ell)}=\underbrace{\Gamma_{a_{1}} \cdot \Gamma_{a_{2}} \ldots \Gamma_{a_{\ell}}}_{\ell}, \quad \ell \geqslant 1 .
$$

In the same way as above we can study the following problem.

$$
(-\Delta)^{m} u(x)=f(x), \quad x \in \Omega, \quad \Gamma_{a_{1}, a_{2}, \ldots, a_{k}}^{(k)}[u](x)=0, \quad x \in \partial \Omega, \quad k=1,2, \ldots, m .
$$

We give a main statement on problem (17) with the proof.

Theorem 2. Let $a_{j}>0, j=1,2, \ldots, m, 0<\lambda<1$ and $f \in C^{\lambda+1}(\bar{\Omega})$. Then problem (17) is uniquely solvable and the solution can be represented as

$$
u(x)=\int_{\Omega} G_{a_{1}}(x, y) f(y) d y
$$

where the Green function $G_{a_{1}}(x, y)$ reads as

$$
G_{a_{1}}(x, y)=G_{D, m}(x, y)+K_{m, n}\left(1-|y|^{2}\right)^{m-1} \int_{0}^{1} s^{a_{1}-1} \frac{\left(1-s^{2}|x|^{2}\right)^{m-1}\left(1-s^{2}|x|^{2}|y|^{2}\right)}{|s x| y\left|-\frac{y}{|y|}\right|^{n}} d s .
$$

\section{BIBLIOGRAPHY}

1. F. Gazzola, H.-Ch. Grunau, S. Guido. Polyharmonic boundary value problems. Springer-Verlag, Berlin (2010).

2. T.S. Kal'menov, D. Suragan. On a new method for constructing the green function of the Dirichlet problem for the polyharmonic equation // Diff. Equat. 48:3, 441-445 (2012).

3. T. Kal'menov, B. Koshanov, M. Nemchenko. Green function representation for the Dirichlet problem of the polyharmonic equation in a sphere // Compl. Variab. Ellip. Equat. 53:2, 177-183 (2008).

4. V.V. Karachik. Green function of the Dirichlet boundary value problem for polyharmonic equation in a ball under polynomial data // Izv. Saratov Univ. (N.S.) Ser. Math. Mech. Inform. 14:4(2), 550-558 (2014). (in Russian). 
5. A. Kumar, R. Prakash. Dirichlet problem for inhomogeneous polyharmonic equation // Compl. Variab. Ellipt. Equat. 53:7, 643-651 (2008).

6. B.Kh. Turmetov. On solvability of a boundary value problem for an inhomogeneous polyharmonic equation with a fractional order boundary operator // Ufimskij Matem. Zhurn. 8:3, 160-175 (2016). [Ufa Math. J. 8:3, 155-170 (2016).]

7. M.A. Sadybekov, B.T. Torebek, B.Kh. Turmetov On an explicit form of the Green function of the Robin problem for the Laplace operator in a circle // Adv. Pure Appl. Math. 6:3, 163-172 (2015).

8. V.V. Karachik, B.Kh. Turmetov. On the Green's function for the third boundary value problem for the poisson equation // Matem. Trudy. 28:1, 17-34 (2018). [Siber. Adv. Math. 29:1, 32-43 (2019).]

9. B.T. Torebek. Green function of Robin boundary value problem in ball // Matem. Zhurn. 15:1, 89-100 (2015). (in Russian).

10. I.I. Bavrin. Operators for harmonic functions and their applications // Diff. Uravn. 21:1, 9-15 (1985). [Diff. Equat. 21:1, 6-10 (1985).]

11. I.I. Bavrin. Integro-differential operators for harmonic functions in convex domains and their applications // Diff. Uravn. 24:9, 1629-1631 (1988). (in Russian).

12. B.Kh. Turmetov, R.R. Ashurov. On solvability of the Neumann boundary value problem for a non-homogeneous polyharmonic equation in a ball // Bound. Val. Probl. 2013:162, 1-15 (2013).

13. A.E. Bekaeva, V.V. Karachik, B.Kh. Turmetov. Solvability of some boundary-value problems for polyharmonic equation with Hadamard-Marchaud boundary operator // Izv. Vyssh. Ucheb. Zaved. Matem. 7, 15-29 (2014). [Russ. Math. Izv. VUZ. Matem. 58:7, 11-24 (2014).]

14. A.V. Bitsadze. Equations of mathematical physics. Nauka, Moscow (1982). [Mir, Moscow (1980).]

Batirkhan Khudajbergenovich Turmetov,

Akhmet Yassawi International Kazakh-Turkish University,

B. Sattarkhanov str. 29,

161200, Turkestan, Kazakhstan

E-mail: turmetovbh@mail.ru 